\title{
Criminologie
}

\section{Le pauvre, la machine judiciaire et la détention}

\section{Jean Hétu}

Volume 9, numéro 1-2, 1976

L’emprisonnement au Québec

URI : https://id.erudit.org/iderudit/017052ar

DOI : https://doi.org/10.7202/017052ar

Aller au sommaire du numéro

Éditeur(s)

Les Presses de l'Université de Montréal

ISSN

0316-0041 (imprimé)

1492-1367 (numérique)

Découvrir la revue

Citer cet article

Hétu, J. (1976). Le pauvre, la machine judiciaire et la détention. Criminologie, 9(1-2), 87-106. https://doi.org/10.7202/017052ar d'utilisation que vous pouvez consulter en ligne.

https://apropos.erudit.org/fr/usagers/politique-dutilisation/ 


\section{LE PAUVRE, LA MACHINE JUDICIAIRE ET LA DÉTENTION}

Jean Hétu

Dans le monde invisible des pauvres, il y en a un certain nombre que l'on ne voit pas parce qu'ils se trouvent derrière les barreaux. On ne peut certes nier le fait que des personnes, à cause principalement de leur condition sociale, soient amenées à commettre certaines infractions, soient plus sujettes que d'autres à être arrêtées, ou trouvent plus difficile d'obtenir leur libération une fois condamnées.

Dans le présent texte, nous aborderons les aspects les plus importants des rapports des pauvres avec le régime d'administration de la justice pénale et nous essayerons de démontrer comment le droit de l'individu à l'égalité devant la loi n'est pas toujours respecté et ce, de façon à frapper plus lourdement les pauvres et les démunis que les riches pour des délits identiques'.

1. Plusieurs des idées énoncées dans ce texte se retrouvent dans J. Hétu et H. Marx. Droit et pauvreté au Québec : Documents, notes et problèmes, Montréal, Les Éditions Thémis, 1974, p. 387 ss. En matière civile, retenons que le nouveau Code de procédure civile déclare à son article 1 que «nonobstant toute disposition contraire d'une loi générale ou spéciale, l'emprisonnement est supprimé en matière civile, sauf le cas d'outrage au tribunals. Cet article oublie cependant de mentionner qu'il existe deux autres cas où la contrainte par corps peut avoir lieu. En effet l'article 668 C.p.c., édicte que «quiconque détériore un immeuble saisi, ou y fait une coupe de bois, est passible d'un emprisonnement d'au' plus six mois*. Nous nous expliquons d'autant plus mal la présence d'un tel article dans notre Code de procédure civile que l'article $397 \mathrm{du}$ Code criminel semble couvrir une telle situation. Quant a l'article 761 C.p.c., il prévoit un emprisonnement d'au plus un an pour toute personne qui contrevient à une injonction. Sur l'état du droit antérieurement au nouveau Code de procédure civile, voir: Acte pour abolir l'emprisonnement pour dette et punir les débiteurs frauduleux dans le Bas.Canada, et pour d'autres objets, 12 Vict., S.C. 1849, c. 42 , et les articles 832 à 852 et 894 à 930 (capias ad respondendum) du Code de procédure civile de 1897. 


\section{A. LA LOI FACE À UNE CULTURE DE PAUVRETÉ}

Bon nombre de pauvres vivent dans ce que Oscar Lewis appelle la «culture de pauvreté"». Cette culture qui peut se définir par tout un ensemble de caractéristiques d'ordre social, économique et psychologique représente a la fois une adaptation et une réaction nécessaires des pauvres à leur position marginale dans notre société:. Pour les classes plus aisées cependant, la structure familiale et les attitudes de ceux qui vivent dans nos quartiers défavorisés ${ }^{4}$ constituent un milieu criminogène plus ou moins latent ${ }^{3}$ que l'on se doit très souvent de réprimer.

Les adultes qui contribuent à la délinquance des mineurs enfreignent l'article 33 de la Loi sur les jeunes délinquants's. Ainsi dans Feeney c. $R$. $^{\dagger}$, le père d'une famille de 12 enfants dont 7 étaient âgés de moins de 18 ans avaient été condamné à la prison par la Cour de bien-être social de Québec. En renversant cette décision, la Cour supérieure affirmait que la Loi sur les jeunes délinquants n'avait pas pour objet d'emprisonner un père de famille qui aurait occasionnellement fait usage de boissons alcooliques ou qui aurait fait usage d'un langage impoli et parfois vulgaire. Les gens n'ont pas tous le même degré d'éducation, la même manière

2. O. Lewis, La Vida, traduit par J. Rosenthal. Paris, Éditions Gallimard, 1969, p. 800. Au Québec, Marie Letellier utilisa ce concept de culture de pauvreté pour décrire une famille du Centre-sud de Montréal dans une recherche intitulée On n'est pas des trous-de-cul, Montréal, Éditions Parti Pris, 1971.

3. Lewis, op. cit., p. 802.

4. La famille de la culture de pauvreté est caractérisée par ale fait que l'enfance n'est pas un stade particulièrement prolongé et protégé de l'existence, une initiation précoce à la sexualité, l'union libre ou le mariage consensuel, un pourcentage relativement élevé d'abandon des femmes et des enfants, une tendance à des familles à forme matriarcale..., une forte prédisposition à l'autoritarisme. l'absence de vie privée...n; Lewis, op. cit., p. 805.

5. Voir par exemple : D. Szabo, «L'inceste en milieu urbainn (1957-1958), L'Année Sociologique, 29. à la p. 52; D. Szabo, Contribution à l'étude de la délinquance sexuelle. Les délits sexuels des adolescents à Montréal, Montréal, Université de Montréal, 1960, p. 45; T. Limoges, Délinquance juvénile et milieux urbains, thèse présentée en vue de lobtention du grade de $\mathrm{Ph}$. D. en Criminologie, Université de Montréal, 1971.

6. S.R.C. 1970 , c. J-3. L'art. 33 porte que : 1) tout adulte qui aaide. induit, engage un enfant à commettre un délit ou tolère qu'il le commette; ou commet quelque acte qui est de nature, tend ou contribue à faire de l'enfant un jeune délinquant ou qui le portera vraisemblablement à le devenir est passible d'une infraction punissable par voie de déclaration sommaire de culpabilité; 2) les parents ou le tuteur de l'enfant qui négligent d'accomplir ce qui tendrait directement à empêcher ledit enfant à être ou à devenir un jeune délinquant, ou de faire disparaître les conditions qui font de lui un jeune délinquant sont passibles d'une infraction punissable par voie de déclaration sommaire de culpabilité.

7. Voir Hétu et Marx, op. cit., p. 164. 
de voir et leur langage varie suivant leurs conditions de vie et, parfois, suivant leur état d'indigence. Mais un père, ajoutait-elle, qui se livrerait à des actes immoraux en présence des enfants, qui blasphémerait d'une façon fréquente, négligerait de surveiller ses enfants ou userait de façon abusive d'alcool pourrait tomber sous le coup de la loi. Mais dans le cas présent la condition de chômeur du pere, concluait le tribunal, ne peut constituer un acte ou une omission de nature à faire de ses enfants mineurs des jeunes délinquants.

Dans La Reine c. $X^{\sharp}$, l'appelante avait été condamnée à 6 mois de prison pour avoir contribué à faire de ses enfants mineurs des jeunes délinquants en vivant en concubinage avec un homme et en négligeant ses responsabilités familiales (elle avait abandonné ses enfants au Bien-être social). Son avocat représenta qu'elle était séparée de corps de son mari, qu'au moment du prononcé de la sentence, elle était enceinte et avait déjà passé un mois de prison, et qu'un cautionnement de garder la paix servirait autant les fins de la justice qu'un emprisonnement de 6 mois. La Cour supérieure rejeta le pourvoi de l'appelante. Elle considéra plutôt que l'emprisonnement était certainement de nature à lui faire apprécier toute l'indignation de sa conduite. Il était temps, disait-on, de se prémunir contre les conséquences économiques engendrées par l'inconduite de certains sujets (les enfants de l'appelante avaient coûté à l'État $\$ 2601.00$ en huit mois) et de protéger les institutions et l'ordre dans notre société. La Cour voyait même dans l'emprisonnement un moyen efficace de lutter contre la délinquance juvénile. Quant au nouveau-né, le tribunal affirmait que l'incarcération de la mère ne pouvait être prejudiciable à l'enfant, vu qu'elle avait dans le passé abandonné ses enfants à la charge de l'État et qu'elle ferait, un jour ou l'autre, probablement la même chose avec son tout dernier.

Une étude menée par deux étudiants en criminologie a également démontré que la majorité des prévenus qui se présentent à la chambre $n^{\circ} 5$ de la Cour municipale de Montréal, communément appelée "cour des relations domestiques", pour répondre à une accusation de refus de pourvoir (art. 197(2) c. cr.) sont des maris sans emploi ou travaillant pour des salaires peu rémunéra-

8. [1968] R.L. 35. Notons que dans $R$. c. $X$., [1969] R.L. 65, il fut jugé que l'adultère continu ou le concubinage n'était pas un crime en soi. Seul est criminel, disait-on, l'adultère qui met en danger les mours des enfants ou rend la demeure du couple adultère impropre à leur présence. 
teurs et vivant dans les quartiers ouvriers de Montréal ${ }^{9}$. La très grande majorité n'en est pas d'ailleurs à son premier contact avec la justice, ayant déjà eu à répondre dans le passé surtout à des accusations de vol, de voies de fait et de conduite en état d'ivresse. On remarque que c'est le Service de bien-être social ou un autre tiers (avocat, parent ou ami) qui est à l'origine de la plainte portée par une assistée sociale contre son "obligé en loi». Qu'il nous suffise de rappeler ici que l'aide sociale peut être refusée, discontinuée, suspendue ou réduite dans le cas de toute personne qui, sans raison suffisante, refuse ou néglige d'exercer les droits et recours qui lui appartiennent ${ }^{10}$. N'est-ce pas là vouloir faire jouer à la cour le rôle d'une agence de recouvrement?

Il est donc assez aisé de comprendre comment le mode de vie des pauvres est de nature à attirer plus facilement l'attention de la justice que celui des autres classes sociales d'autant plus que les quartiers défavorisés bénéficient habituellement d'une surveillance accrue de la part de la police. La Commission de réforme du droit a d'ailleurs établi que «les jeunes en chômage ou détenant un emploi peu rémunérateur constituent la majeure partie des personnes qui comparaissent en correctionnelle sous les accusations de vol, de voies de fait ou d'inconduite "', Il y a certes une relation à faire entre les crimes dont les tribunaux sont le plus souvent saisis, soit vol et recel (la valeur moyenne des biens volés étant en général inférieure à \$200), infractions concernant les véhiculesautomobiles, ivresse ou le fait de troubler la paix publique, voies de fait, vol avec effraction, fraude, et le statut social des prévenus ${ }^{12}$.

On ne peut nier qu'un certain nombre de pauvres, en raison principalement de leur situation économique, sont souvent amenés un peu par la force des choses à commettre certaines infractions criminelles. Ainsi par exemple dans $R$. c. Gauthier ${ }^{13}$, un père de 11 enfants qui savait tout juste signer son nom (lorsqu'il réclamait du Bien-être social, une autre personne remplissait les formules) et qui avait eu recours au Bien-être social pour subvenir aux besoins

9. Voir J. Hétu et H. Marx, "Droit et pauvreté, Chronique régulièren (1974), $34 R$, du B., 254.

10. Loi de l'aide sociale, L.Q. 1969, c. 63, art. 12.

11. Commission de réforme du droit, les Principes de la détermination de la peine et du prononcé de la sentence, Document de travail 3, Ottawa, Information Canada, mars 1974, p. 8.

12. Id., p. 7. Rousseau).

13. Cour des sessions de la paix, Montréal, nº 73-9397, 7 mai 1974 (J. 
de sa famille entre 1969 et 1973 plaida coupable à une accusation de fraude de $\$ 2000$ du ministère des Affaires sociales (art. 338 C. cr.). Trouvant très difficile de rendre une sentence dans un tel cas, le tribunal disait : «Si la Cour envoie le prévenu pour une période prolongée en prison, elle replace de nouveau toute la famille sur le Bien-être social, par contre une sentence légère serait de nature à favoriser la prolifération des infractions de ce genre $^{14} \mathrm{n}$. Ayant bien réfléchi sur cette affaire, la Cour condamnait le prévenu à purger dix jours de prison d'une manière discontinue du samedi 18 hres au dimanche 17 hres, à garder la paix pendant deux ans, à avertir de tout changement d'adresse et à rembourser la somme de $\$ 2000$ dans un délai de deux ans. Notre société de consommation (ou de convoitise) avec tous ses libres services a probablement sa part de responsabilité. Les mass media nous rapportent fréquemment le cas de ménagères qui, n'ayant pas les moyens de se payer tel ou tel bien de consommation, n'ont pu résister à la tentation de se le procurer quand même et ont été trouvées coupables de vol à l'étalage ${ }^{1 .}$. Certains témoignages devant la Commission d'enquête sur le crime organisé nous révélaient récemment comment les prêteurs sur gages et les usuriers locaux forcent leurs clients déshérités à passer de la drogue, de la cocaïne ou des faux billets de banque pour payer leurs dettes ${ }^{16}$.

Ce n'est pas une révélation non plus d'affirmer que la pauvreté et le chômage sont des facteurs importants à l'origine de la prostitution. Dans La Reine c. Gallant ${ }^{17}$, un couple arrivait difficilement à boucler son budget avec les prestations de bien-être social et la femme faisait un petit surplus grâce à la prostitution. Le tribunal trouva alors son compagnon coupable d'avoir vécu des fruits de la prostitution tout en soulignant que ce dernier et sa compagne s'étaient, par le fait même, "montrés indignes d'être bénéficiaires d'allocations du bien-être social ${ }^{18}$.

La Commission de réforme du droit du Canada a fait ces dernières années des efforts louables pour éliminer certains «anachronismes" de notre droit pénal. Il n'en demeure pas moins qu'on

14. Id., p. 3.

15. Voir par exemple: «Vol à l'étalage : prison pour une assistée sociale», la Presse, 20 août 1975, p. A-11.

16. Le Devoir, 26 septembre 1974, p. 3.

17. [1969] R.L. 419 conf. par [1969] B.R. 900. Pour un cas plus récent de prostitution par nécessité. voir la Presse, 22 août 1974, p. A-6.

18. [1969] R.L. 423. 
y retrouve encore, à tort ou à raison, des dispositions de nature socio-économique.

Plusieurs articles, tel celui qui faisait du vagabondage défini comme le fait pour une personne d'être trouvée sans aucun moyen apparent de subsistance ou de mendier une infraction pénale ${ }^{19}$, ont en effet été éliminés du Code criminel. On n'en constate pas moins cependant que l'on peut encore assez facilement aujourd'hui inculper quelqu'un pour de semblables infractions en vertu d'autres articles du Code criminel, tel par exemple celui relatif à la flânerie et au tapage dans un endroit public ${ }^{20}$, ou de règlements municipaux $^{21}$. C'est ainsi, par exemple, que l'action que mène la police contre les 1500 clochards de Montréal résulte dans près de 5000 arrestations annuellement ${ }^{22}$. En 1971, la Old Brewery Mission de Montréal estimait que $46 \%$ des clochards avaient un dossier judiciaire $^{23}$.

D'autres dispositions de nature économique, telles celles relatives au refus de pourvoir ${ }^{24}$ ou à l'obtention par fraude d'aliments, de logement ou d'autres commodités ${ }^{25}$ demeurent et perpétuent en quelque sorte le rôle d'agence de recouvrement de nos tribunaux.

\section{B. LA DISCRÉTION DE LA POLICE ET DE LA COURONNE}

Nous avons vu jusqu'ici comment notre doit pénal était loin de coller à la réalité familiale des milieux défavorisés et comment les gens qui y vivent sont amenés, un peu par la force des choses, à enfreindre nos lois.

Parmi ceux qui commettent des infractions, tous ne sont pas arrêtés et un nombre encore moindre est poursuivi. Les officiers

19. Ancien article 164 C. cr. Voir $R$. c. Hawkins (1971), 2 C.C.C. (2d) 291.

20. Art. 171 C. cr.

21. Voir : Règlement pour empêcher que les piétons ou la circulation ne soient entravés et la paix publique troublee dans les rues, ruelles et places publiques de la Cité, $n^{\circ} 333$, Ville de Montréal; Règlement concernant les collectes publiques (tag-days), les autres collectes pour fins charitables et la mendicité, $\mathrm{n}^{\circ} 1447$, Ville de Montréal; Ordre public dans le métro, $n^{\circ} 3361$, Ville de Montréal.

22. M. Magnant et al., Étude sur les clochards, Étude financée grâce à un octroi de Perspective-Jeunesse, 1973, p. 47.

23. $I d$, p. $2 \mathrm{t}$.

24. Art. 197 C. cr.

25. Art. 332 C. cr. 
de police et les avocats de la Couronne ont en effet un pouvoir discrétionnaire étendu sur l'arrestation, le choix ou le retrait des accusations, de même que sur la suspension des poursuites et la négociation des plaidoyers. Or, encore ici, les pauvres apparaissent plus pénalisés que les riches. Comme le souligne la Commission de réforme du droit : «Le pouvoir discrétionnaire existant dans la mise en application de la loi tend à soustraire les commerçants et les professionnels aux tribunaux criminels. Les fraudes ou les vols commerciaux peuvent souvent faire l'objet d'un règlement privé ou d'une restitution. D'autre part, les personnes démunies d'argent ou d'influence, qui sont arrêtées pour avoir commis un larcin ou un vol à l'étalage, ne se voient pas offrir l'occasion de réparer le préjudice qu'elles ont causé, et nombreuses sont celles qui sont immédiatement citées devant les tribunaux. Il arrive souvent que ces citoyens ordinaires n'aient pas le prestige suffisant ni les possibilités qui leur permettent de négocier ou de faire appel aux ressources psychiatriques, éducatives ou économiques, de façon à effectuer un règlement et à s'éviter ainsi un procè ${ }^{26}{ }^{26}$.

L'on sait que les enfants des quartiers à faible revenu sont plus souvent arrêtés et envoyés devant la Cour de bien-être social sous prétexte, entre autres, que leurs parents ne s'occupent pas d'eux alors que les enfants des classes plus aisées, et dont la police connaît souvent la famille, se verront plutôt donner un simple avertissement pour des délits identiques. Comme certains s'amusent à le dire, si l'on n'a pas d'argent, que l'on est pauvrement vêtu et que l'on est recueilli ivre dans la rue, on est un "robineux»; mais si l'on est habillé d'une chemise et d'une cravate et que l'on est bien rasé tout en étant ivre dans la rue, on est plutôt un alcoolique que l'on retournera en taxi chez soi. De même, si l'on est surpris à voler à l'étalage et que l'on est bien nanti, on serait un kleptomane; si l'on n'a pas d'argent, on serait plutôt un voleur. De fait, il a été démontré aux États-Unis que des considérations de condition sociale ainsi que le facteur racial influaient considérablement sur le pouvoir discrétionnaire d'arrestation de la police ${ }^{27}$. Au Canada, surtout dans les provinces de l'Ouest où la majorité des détenus

26. Commission de réforme du droit, op. cit, supra, note 13 p. 8.

27. Voir : P. Wald, «Poverty and Criminal Justice», dans N. Johnston, L. Savitz et M.E. Wolfgang. The Sociology of Punishment and Correction, $2+$ ed., New York, Toronto, Wiley \& Sons Inc., 1970, p. 271 à 273; C. Baker Motley, aLaw and Order and the Criminal Justice System», [1973], 64 The Journal of Criminal Law and Criminology, 259. 
sont des Indiens ou des Métis ${ }^{2 \mu}$, on est en droit de soulever des hypothèses semblables.

De plus on constate dans les deux pays que la police est beaucoup plus sélective dans son pouvoir d'arrestation tout dépendant qu'il s'agisse d'un quartier huppé ou des «zones grises» de la ville. Il arrivera en effet très rarement pour ne pas dire jamais que des pauvres, en raison principalement de ce fatalisme qui les caractérise, poursuivent des policiers pour arrestation illégale, d'où la probabilité plus grande de certains abus.

Au niveau de la mise en accusation, plusieurs délits peuvent être poursuivis soit comme actes criminels soit par voie de déclaration sommaire de culpabilité. Qu'il nous suffise ici de mentionner ceux relatifs au refus de fournir les choses nécessaires à la vie ${ }^{29}$, à l'obscénité ${ }^{30}$, à un véhicule à moteur ${ }^{31}$ et aux voies de fait simples ${ }^{32}$. Le procureur général et les avocats de la Couronne ont entière discrétion pour déterminer le mode de poursuite et ce choix affectera nécessairement le casier judiciaire du prévenu et, éventuellement, toute demande future de pardon ${ }^{33}$.

La Cour Suprême du Canada s'est déjà penchée sur ce problème et a décidé que le pouvoir discrétionnaire de la Couronne de mener les procédures criminelles comme elle le jugeait bon ne violait pas le principe du droit de l'individu à l'égalité devant la loi et à la protection de la loi qu'on retrouve dans la Déclaration canadienne des droits ${ }^{34}$ et qu'il faisait partie, à la date de l'entrée en vigueur de la Déclaration, de la conception britannique et canadienne de l'égalité devant la loi ${ }^{33}$. Le tribunal dans cette décision était d'opinion que la loi contestée, qui permettait un choix quant au mode de poursuite, n'établissait en soi aucune distinction entre une personne ou classe de personnes particulières et quelque

28. Voir : D.A. Schmeiser, H.W.B. Heumann et J.R. Manning. la Délinquance chez les autochtones et la loi, (préparé pour la Commission de réforme du droit du Canada), Ottawa, Information Canada, 1974; Rapport du Comité canadien de la réforme pénale et correctionnelle, Ottawa Imprimeur de la Reine, 1969, p. 442; The Canadian Corrections Association, Indians and the Law, Ottawa, the Canadian Welfare Council, 1967, p. 33 ss.

29. Art. 197 C. cr.

30. Art. 159 et $165 \mathrm{C}$. cr.

31. Art. 233 (1) (2) (4), 238 (3), 239 C. cr.

32. Art. 245 (1) C. cr.

33. Voir : Loi sur le casier judiciaire, S.R.C. 1970, 1+r Supp., c-12.

34. S.R.C. 1970, App. III.

35. Smythe c. La Reine [1971], R.C.S. 680. 
autre membre de la société et que ses dispositions, qui s'appliquaient assurément sans distinction à tout le monde, conféraient simplement au Procureur général du Canada le pouvoir de décider, selon son propre jugement et dans tous les cas, le mode de poursuite des infractions décrites dans la $\mathbf{l o i}^{36}$. Certaines considérations pratiques comme la peur de paralyser l'administration de la justice n'étaient pas sans influencer la décision de notre Cour Suprême. Il serait impossible, soulignait-elle, d'appliquer la loi si un certain pouvoir discrétionnaire n'était dévolu à une personne ayant autorité ${ }^{37}$. De plus, ajoutait-elle, si la prétention de discrimination de l'appelant était accueillie, les quelques trente articles du Code criminel et les articles de quelque quarante lois canadiennes où le pouvoir de choisir de procéder par voie de déclaration sommaire de culpabilité ou par voie de mise en accusation est conféré, deviendraient inopérants ${ }^{38}$.

Même si l'élimination complète du pouvoir discrétionnaire de la police et de la Couronne ne favoriserait probablement pas les fins de la justice, il nous est permis d'espérer cependant que des cas analogues soient traités, autant que possible, de la même façon. Dans cette optique, nous croyons souhaitable d'en arriver un jour à des mécanismes de contrôle efficaces de cette discrétion car, après tout, discrétion n'a jamais voulu dire discrimination ${ }^{39}$.

\section{LA REFORME DU CAUTIONNEMENT}

Il n'y a pas encore si longtemps, nos tribunaux exigeaient de façon générale un cautionnement, en argent ou sur immeuble, afin de s'assurer de la présence d'un accusé à son procès. Il est évident qu'avec une telle façon de procéder le citoyen, pauvre, arrêté pour ivresse au volant, vagabondage ou refus de pourvoir, se voyait fréquemment privé de sa liberté faute de solvabilité. La liberté du prévenu dépendait moins de la nature de l'accusation que de l'importance de ses ressources financières.

La Commission d'enquête sur l'administration de la justice en matière criminelle et pénale au Québec a déja souligné à quel

36. Id., p. 685 .

37. Ibid.

38. Id., p. 687. Pour une critique de cet arrêt, voir : F. Chevrette et H. Marx, "Libertés publiques. Chronique régulière" (1972), 32 R. du B. 64.

39. Voir dans ce sens le Rapport du Comité canadien de la réforme pénale et correctionnelle, supra, note 28 , p. 46 ss. 
point la libération d'un individu avant le procès avait d'influence sur la sentence :

À toutes fins pratiques, la liberté avant le procès constitue, en effet, une sorte de test ou de probation dont les juges ne peuvent que tenir compte. Celui qui, pendant les mois séparant l'arrestation du procès, s'est comporté de façon louable, a prouvé en somme qu'il pouvait faire bon usage de sa liberté et se réhabiliter par voie d'amende ou de probation.

Par contre, lorsque le procureur de la Couronne et le magistrat de la première comparution ont tous deux jugé l'individu inapte à une liberté avant le procès, le juge du procès lui-même s'en trouve conditionné et il hésite davantage à faire usage de l'amende ou de la sentence suspendue. Malheureusement, l'incapacité de fournir un cautionnement aboutit souvent aux mêmes conséquences, parce qu'elle empêche l'accusé de faire la démonstration de ses bonnes dispositions ${ }^{40}$.

Le fait que l'accusé ait passé quelques nuits en prison avant son procès n'est pas de nature, d'ailleurs, à l'encourager psychologiquement à plaider sa cause. Enfin, si l'accusation est retirée par manque de preuves, le pauvre a quand même subi un châtiment réel alors qu'il n'a commis aucun crime"'.

La Partie XIV du Code criminel fut remplacée assez récemment par la Loi sur la réforme du cautionnement ${ }^{+2}$ qui libéralise la procédure de mise en liberté provisoire des personnes arrêtées ou inculpées d'avoir commis une infraction criminelle. Selon cette loi, la liberté provisoire n'est plus un privilège et devient la règle. La détention d'un prévenu n'est justifiée que si elle est nécessaire pour assurer sa présence devant le tribunal, dans l'intérêt ou pour la protection de la sécurité du public. En fait, si la Couronne ne s'oppose pas à la mise en liberté sans condition du prévenu, le juge doit le remettre en liberté sans que ce dernier ait à la demander. D'autre part, si le poursuivant s'y oppose, il doit établir ses motifs par une preuve prépondérante.

40. La Société face au crime. Principes fondamentaux d'une nouvelle action sociale, vol. 1, Québec, 1968. p. 41 .

41. Sénat du Canada, la Pauvreté au Canada, Ottawa. Imprimeur de la Reine, 1971, p. 152.

42. S.R.C. 1970, 2" Supp., c. 2 ou S.C. 1971, c. 37. Sanctionnée le 19 mars 1971, cette loi est entrée en vigueur le 3 janvier 1972, voir: Gazette du Canada, Partie II, Vol. 105, $\mathrm{n}^{\circ}$ 23, DORS/71-611. Voir aussi : A. Dumontier, «La loi sur la réforme du cautionnement" (1972), 13 C. de D. 79; J.A. Scollin, The Bail Reform Act. Toronto, Carswell, 1972; G. Brassard, "La liberté provisoire en attendant la décision d'un appeln (1972), 7 R.J.T. 495. 
La nouvelle loi a de plus presque éliminé, à toutes fins pratiques, le cautionnement en argent qui permettait trop souvent aux professionnels du crime d'acheter leur liberté provisoire tout en pénalisant les défavorisés. Comme le soulignait judicieusement le tribunal dans Powers c. $R^{43}$ :

The lack of financial means of the accused or his prospective bondsmen and sometimes the unfortunately long periods while awaiting trial, resulted in detention of persons who, in themselves, did not necessarily represent any threat to public safety and who also would probably be available for their trial. It is now generally recognized that the purposes of the criminal law should be carried out without unwarranted interference with the freedom of the individuals of society...

Malgré tout il n'en demeure pas moins que si une personne défavorisée, parce qu'elle est sans adresse comme cela arrive fréquemment en ce qui concerne les clochards, est arrêtée pour une infraction pénale, elle devra demeurer derrière les barreaux afin d'assurer sa présence devant le tribunal.

\section{LA DISPARITÉ DES SENTENCES}

Problème délicat qu'est celui de la disparité dans les sentences. Il peut en effet arriver que l'on ait l'impression que nos tribunaux imposent des sentences très différentes pour de semblables infractions. D'aucuns considèrent, à juste titre sans doute, que la disparité des sentences est non seulement acceptable mais souhaitable dans l'optique de l'individualisation des peines. Il est fort possible que deux personnes trouvées coupables de cambriolage ne représentent pas le même danger pour la société et que l'une d'elle puisse bénéficier de la clémence du juge alors que l'autre soit condamnée à quelques années de prison. Mais le milieu familial et social n'est-il pas encore ici de nature à influer sur la détermination de la sentence? Le rapport présentence n'en fait-il pas d'ailleurs mention? Que faut-il penser, par exemple, de l'application du critère de l'homme raisonnable, qui est avant tout un concept de classe moyenne et bourgeoise, comme facteur de mitigation de la peine?

Aux États-Unis, il a été maintes fois démontré que les «Lower Socio-Economic Strata» et plus particulièrement les noirs étaient ceux qui purgeaient les plus longues sentences et que l'on trouvait 
le plus souvent dans les institutions pénitenciaires ${ }^{+4}$. Au Canada, l'on constate qu'un pourcentage anormal d'autochtones ont été trouvés coupables d'infractions et condamnés à des peines d'emprisonnement, qu'ils ont plus fréquemment un casier judiciaire et en général plus chargé que les non-autochtones et enfin qu'ils ont moins de chance d'obtenir une mise en liberté surveillée ou de bénéficier d'un rapport pré-sentenciel ${ }^{45}$.

D'autre part il n'est pas rare de voir des avocats de la défense plaider que leur client appartient à une famille honorable (déjà assez humiliée), qu'il a reçu une excellente éducation, et que, vu son statut social et les dommages subis à sa réputation, il a été suffisamment puni sans que le juge ait besoin d'ajouter d'autres peines. Si de telles représentations peuvent parfois porter fruits, la règle n'en demeure pas moins que celui «qui a reçu une formation irréprochable a moins d'excuses pour commettre un crime que le jeune qui a vécu dans un milieu crapuleux de parents qui l'ont abandonné ou parfois poussé vers le crime $\mathrm{e}^{44} \mathrm{~m}$. Comme le soulignait le juge dans La Reine c. Jacques St-Germain ${ }^{47}$ : "A la suite du Fabuliste, une large fraction de l'opinion publique est depuis toujours, même si c'est inexact, portée à croire que la Justice a tendance à être plus tendre pour les puissants que pour les faibles. Le Tribunal doit veiller à ne pas donner prise à cette critique, mais doit se garder de l'effet contraire. Après avoir tenu compte de toutes les circonstances, le Tribunal doit se montrer aussi sévère envers un médecin, un ingénieur, un comptable, un avocat ou un notaire qu'envers un ouvrier, mais pas plus. Il importe de rappeler que la Justice et la Loi ne doivent jamais faire acception des personnes.» Mais le même juge, après avoir constaté que l'accusé (un médecin) par son témoignage et sa façon d'agir avait indiqué

44. Voir : H.A. Bullock, «Significance of the Racial Factor in the Length of Prison Sentences" (1961), 52 The Journal of Criminal Law, Criminology and Police Science, 411: T.P. Thomberry, aRace. Socioeconomic Status and Sentencing in the Juvenile System" (1973), 64 The Journal of Criminal Law and Criminology 90; Baker Motley, loc. cit., p. 259. a have found, affirmait le Dr F. Wertham dans le New York Law Journal du 19 juillet 1974, that in actual practice the rich man is apt to be treated as neurotic whereas the poor man is labelled schizophrenic; but when a crime is committed the situation is reversed: the rich man is exculpated as schizophrenic while the poor man is dealt with as just neurotic.n

45. Voir supra, note 28.

46. A. Rivard, «Les sentences en droit pénal» (1969), $29 R$. du B. 167, aux p. 171 et 176; G. Robert, «Problème du sentencing : vol à l'étalage» (1970), 30 R. $d u$ B. 220 , à la p. 223. Voir aussi : R. c. Millen (1973), 11 C.C.C. (2d) 70 oủ un jeune homme de 20 ans, athe product of a family plagued by poverty", était libéré inconditionnellement après avoir été trouvé coupable d'ivresse au volant.

47. Cour des sessions, Montréal, $n^{\circ} 73-1472,25$ mars 1974, 5 (J. Roche) 
un état d'esprit peu préparé à une réhabilitation prochaine, condamnait ce dernier à une année de prison pour négligence criminelle. Etait-ce faire exception des personnes? Nous nous le demandons.

Dans une décision majoritaire ( 2 contre 1 ), la Cour d'appel du Québec maintenait récemment la condamnation d'un notaire de Montréal à deux ans de prison pour avoir détourné une somme de près de $\$ 40000$ aux dépens de trois de ses clients. «Sa formation, sa connaissance de la loi lui permettent donc d'apprécier la gravité d'un abus de confiance, et les devoirs de sa charge l'astreignent à une éthique sévère», ajoutait-on. Et la Cour de continuer : "Le rang social qu'il occupe ne peut donc être un facteur de mitigation de sentence. Il l'obligeait plutôt à donner l'exemple du respect des lois et, dans ces circonstances, la sentence imposée ne paraît que juste et raisonnable.» Un autre juge était plutôt d'avis qu'une période de probation de deux années, en liberté, serait un châtiment suffisant, suite aux nombreux inconvénients qui ont valu au prévenu la perte du droit d'exercice de sa profession ${ }^{48}$.

Notons de plus que jusqu'à tout récemment les pauvres étaient plutôt mal défendus devant les tribunaux de justice pénale. Un certain nombre n'était même pas représenté et plaidait coupable de façon à en finir le plus rapidement possible avec la justice. Dans un tel contexte il était assez compréhensible de retrouver plus de défavorisés que de bien nantis dans nos prisons ${ }^{49}$. Aujourd'hui l'instauration d'un système de cliniques juridiques dévouées aux intérêts des économiquement faibles a sensiblement modifié cet état de choses ${ }^{50}$. Les avocats ne sont-ils pas passer maître dans l'art de jouer avec la procédure de façon à mettre toutes les chances du côté de leur client et ainsi lui éviter la prison, ou du moins en retarder l'échéance? À ce propos un incident récent nous laisse perplexe. Au mois de février 1973, les avocats de l'Assistance judiciaire de Montréal se plaignaient qu'un juge de la Cour des sessions de la paix soit saisi exclusivement des causes de leurs clients parce que les avocats de pratique privée refusaient de plaider devant lui. Après avoir reconnu que personne ne pouvait avoir le choix de son juge et nié le fait que ce juge soit "réservé» presque exclusivement à un groupe particulier de citoyens, le juge en chef n'en décidait pas moins de modifier le rôle de ce juge

48. La Presse, 25 septembre 1973. p. A-8.

49. Dans le même sens voir : Wald, loc. cit., p. 393.

50. Loi de l'aide juridique, L-Q. 1972, c. 14. 
pour y inclure des causes de pratique privée. Au mois d'avril 1973, le Barreau du Québec adoptait une résolution enjoignant aux avocats de la province de ne plus se présenter devant ce juge et le ministre de la Justice intervint pour le forcer à prendre sa retraite". De tels incidents nous portent à croire qu'il y a deux types de justice. Quoi qu'il en soit, rappelons-nous qu'il ne suffit pas que justice soit rendue, il faut encore que l'on ait limpression qu'elle est rendue.

\section{E. LES AMENDES}

Quiconque est trouvé coupable d'une infraction à un règlement municipal à Montréal est passible d'une amende, avec ou sans frais, ne devant pas excéder $\$ 100$ et à défaut, d'un emprisonnement maximum de 60 jours $^{52}$. Toute personne déclarée coupable d'une infraction punissable sur déclaration sommaire de culpabilité est passible d'une amende d'au plus $\$ 500$ ou d'un emprisonnement de 6 mois $^{53}$. Lorsqu'il s'agit d'un acte criminel, les peines sont encore sévères ${ }^{54}$. Pour plusieurs de telles amendes ne sont que de simples "coûts de production" ajoutés au passif de leurs activités illégales" Pour les pauvres, ces peines pécuniaires, même minimes, représentent un fardeau financier beaucoup plus important. L'emprisonnement avec toutes les conséquences qui en découlent (casier judiciaire, perte d'emploi, difficultés financières accrues pour la famille, augmentation des prestations de bien-être social, troubles émotifs et familiaux) apparaît, encore ici, attribuable davantage au manque d'argent qu'à la conduite criminelle. Tout cela, «c'est indirectement

51. Hétu et Marx. op. cit., supra, note 1, p. 413.

52. Sanctions pour infractions aux règlements, $\mathrm{n}^{\circ} 4029$ tel que modifié par le règlement $n^{\circ} 4172$, Ville de Montréal, art. 1.

53. Art. $722 \mathrm{C}$. cr.

54. Art. 645 (3) (4), 646 et $650 \mathrm{C}$. cr. Règle générale, un accusé déclaré coupable d'un acte criminel punissable d'un emprisonnement de 5 ans ou moins peut être condamné simplement à une amende sauf s'il s'agit d'une infraction punissable d'une période minimum d'emprisonnement; lorsqu'il s'agit d'un acte criminel punissable d'un emprisonnement de plus de 5 ans, le juge peut condamner l'accusé à une amende en sus, mais non au lieu de l'emprisonnement.

55. Voir $R$ c. Johnson (1972), 5 C.C.C. (2d) 541 où le tribunal de première instance avait condamné "a man of means" à 7 jours de prison pour ivresse au volant en disant que l'imposition d'une amende ne pouvait lui servir de leçon. Le livre blanc sur la justice déposé récemment par le ministre de la Justice contient une recommandation proposant que les amendes soient proportionnées aux revenus du coupable d'une infraction, voir J. Choquette, la Justice contemporaine, Québec, Éditeur officiel du Québec, 1975, p. 249-250. 
le résultat du délit et directement, c'est le résultat d'être pauvre et incapable de payer l'amende ${ }^{56} n$.

Dans Regina c. Natral"i, le défendeur avait été reconnu coupable d'avoir conduit un véhicule-automobile en état d'ivresse et condamné le 14 juillet 1971 à $\$ 250$ d'amende et, à défaut, à 15 jours de prison, en plus de voir son permis de conduire suspendu pour 6 mois. La moitié de l'amende devenait payable le 16 août 1971 et l'autre $\$ 125$, le 14 septembre 1971 . Même après avoir obtenu un délai supplémentaire de paiement, le défendeur se dit incapable de payer son amende. Il invoqua alors devant la Cour d'appel de la Colombie-Britannique le fait que l'article 722 du Code criminel relatif à l'emprisonnement à défaut de paiement d'une amende allait à l'encontre des articles 1(b), 2(a) et 2(b) de la Déclaration canadienne des droits. Ces articles visent «le droit de l'individu à la protection de la loi», «l'emprisonnement arbitraire», et les «peines ou traitements cruels ou inusités». En d'autres mots, on soutenait que «the routine imposition of a fine with imprisonment in default of payment places rich and poor on an unequal footing before the law, in that the one class has the means to pay and the ability to purchase its liberty whereas the other class is not in that position. So, it is said, we have a situation where the right to equality before the law is violated" is.

Après avoir énoncé que l'article $722 \mathrm{C}$. cr. prévoyait non pas un emprisonnement de routine mais plutôt un examen des moyens financiers du coupable avant de le condamner à une amendein,

56. Sénat du Canada, op. cit., p. 153. Entre le $l^{\text {rr }}$ octobre 1972 et le l'*r octobre 1973, 47,7\% des détenus de la prison de Bordeaux purgeaient une peine d'emprisonnement comme substitut à une amende non payée à la suite surtout de délits d'ivresse, de "flânage", de mendicité ou d'infractions au code de la route. Ces condamnations correspondaient presque toutes à des amendes de moins de $\$ 60$. Enfin $65 \%$ des amendes non payées rèsultaient en des peines de moins de 15 jours de prison. Voir C. Menghile et R. Pépin, Description de la population de l'établissement de détention de Montréal, École de criminologie. Université de Montréal, juin 1974: J. Hétu et H. Marx, «Les amendes, lemprisonnement et la pauvretén (1974), 34 R. du B. 575 .

57. (1973), 32 D.L.R. (3 ${ }^{\mathrm{d}}$ ). 241 (Cour d'Appel de la C.-B.).

58. Id., p. 248.

59. Art. 722 C. cr. (1) Sauf lorsque la loi prévoit autrement de façon expresse, toute personne déclarée coupable d'une infraction punissable sur déclaration sommaire de culpabilité est passible d'une amende d'au plus cinq cents dollars ou d'un emprisonnement de six mois, ou des deux peines à la fois. ... (3) Une cour des poursuites sommaires peut ordonner, sous réserve des dispositions du présent article, qu'une amende rendue payable par un arrêt a) soit versée sur-le-champ, ou b) soit versée à l'époque et aux conditions que la cour des poursuites sommaires peut fixer. (4) Lorsqu'une cour des poursuites sommaires ordonne à un accusé de payer une amende, la cour ne doit pas, au moment de l'imposition de la sentence, ordonner 
la Cour d'appel décida majoritairement "there has not here been any abrogation, abridgement or infringement of the appellant's right to equality before the law. In being subjected to imprisonment in default of payment of the fine imposed, the appellant has not been treated differently to others. A poor man who is fined inevitably suffers more than a rich man. Some persons are more sensitive that others and for a variety of reasons may suffer from confinement far more than others. Cultural background, personnal environment and character may all play their part... Equality before the law has no relation to such matters and things as these ${ }^{50}$.o

Quant au juge dissident, il en venait à la conclusion que l'appelant n'avait véritablement pas les moyens financiers de payer l'amẹnde, que le juge de première instance le savait, et que la sentence équivalait, à toutes fins pratiques, à un emprisonnement certain. Il considérait plus appropriée une condamnation à une amende de $\$ 250$ sans emprisonnement à défaut de paiement, payable en versements mensuels de $\$ 20$ à partir du premier décembre 1971. La Couronne pourra toujours, le cas échéant, utiliser son recours civil en recouvrement d'une peine pécuniaire prévue à l'article $652 \mathrm{C}$. cr., ajoutait-il ${ }^{\mathrm{l} 1}$.

La Cour d'appel du Québec tenait récemment les propos suivants concernant les sentences pour vol à l'étalage : "The crime of shoplifting has become so widespread that exemplary sentences may be required. I $[M$. le juge Montgomery] cannot agree that a first offender in such a case should automatically receive a suspended sentence nor that an offender should escape punishment simply because he does not have the means to pay a fine ${ }^{(i 2)}$. A notre avis il ne

le paiement immédiat de l'amende, sauf a) si elle est convaincue que la personne condamnée dispose de moyens suffisants pour lui permettre de payer l'amende sur-le-champ, b) si. lorsque la cour demande à la personne condamnée si elle désire un délai de paiement, cette dernière ne sollicite pas ce délai, ou c) si, pour tout motif spécial, la cour estime opportun qu'il ne soit accordé aucun délai.... (9) Quant il apparaît à la cour qu'une personne qui s'est vu accorder un délai de paiement est âgée d'au moins seize ans et d'au plus vingt et un ans, la cour doit, avant d'émettre un mandat ordonnant l'incarcération de ladite personne à défaut du paiement de l'amende. obtenir et étudier un rapport sur la conduite et la capacité de paiement de l'accusé. (10) Lorsqu'un délai de paiement a été accordé sous le régime du paragraphe (3), la cour qui a infligé la sentence peut, sur demande faite par l'accusé ou en son nom, accorder un délai supplémentaire de paiement.

60. $R$ c. Natrall (1973), 32 D.L.R. (34), 241, à la p. 250.

61. Id., p. 255.

62. Voir : Inge Hollinger c. Her Majesty the Queen, et Patrick Henry c. Her Majesty the Queen, C.A. Montréal, n 10-000139-73. II octobre 1973; Sa Majesté la Reine c. Louise Adam, C.A. Montréal, n 10-000007-748, 3 décembre 1974 (JJ. Rinfret, Bernier et Mayrand) 
s'agit pas de permettre à un groupe social d'échapper à toute punition mais bien d'éviter qu'une partie de notre population soit pénalisée davantage que d'autres citoyens. Nous croyons qu'il est tout à fait inacceptable que nos prisons servent principalement à incarcérer tous ceux incapables de payer une amende ${ }^{633}$.

Le fait que l'appelant dans Natrall ait vu son permis de conduire suspendu pour une période de six mois ne constituait-il pas une peine suffisante? L'instauration d'un système de points de démérite en matière d'infractions routières et la suspension du permis de conduire qui peut en découler n'a-t-il pas enlevé toute justification à l'imposition d'une amende? À moins que l'État soit motivé par des considérations purement financières. Quoi qu'il en soit l'emprisonnement à défaut de paiement des amendes coûte très cher à l'État et détruit la fonction de dissuasion de la prison ${ }^{64}$. Aussi des efforts devraient être faits afin de trouver d'autres formes de sanction moins discriminatoires à l'égard des pauvres (e.g. participation à des programmes de formation technique, procédures civiles de saisie à défaut de paiement d'une amende, etc. $)^{6.5}$.

\section{F. ORDONNANCE DE LIBERATION, SURSIS DU PRONONCÉ DE LA SENTENCE ET LIBÉRATION CONDITIONNELLE}

Lorsqu'un accusé est reconnu coupable d'une infraction criminelle, le tribunal peut au lieu de condamner l'accusé, prescrire par ordonnance qu'il soit libéré inconditionnellement ou aux conditions prescrites dans une ordonnance de probation s'il considère qu'une telle mesure est dans l'intérêt véritable de l'accusé sans nuire à l'intérêt publicicis. L'accusé n'est pas alors censé avoir été déclaré coupable de l'infraction même si l'infraction est portée à son casier judiciaire. Ce dernier peut donc affirmer qu'il n'a pas été trouvé coupable de telle infraction mais il n'en va pas de même si on lui demande s'il a un casier judiciaire. Dans d'autres cas,

63. $\dot{A}$ ce sujet voir Schmeiser. Heumann et Manning, loc. cit., ainsi que Menghile et Pépin, op. cit.

64. Schmeiser, Heumann et Manning, loc. cit., p. 71 et 73.

65. Voir : Id., p. 73; Commission de réforme du droit du Canada, supra, note 13. L'art 663 (1) $\mathrm{c}$ du C. cr. permet l'emprisonnement pendant les fins de semaine sans interrompre le travail habituel de la personne condamnée. Voir aussi : L. Kosrabcewicz-Zubkowski, aLes mesures prevves en remplacement des courtes et moyennes peines privatives de liberté au Canada», (1973), 8 R.J.T. 305.

66. Art. 662 (l) C. cr. Voir : J.-C. Hébert, «L'inévitable casier judiciaire» (1973), 4 R. du B. 390. 
le tribunal pourra, vu l'âge et la réputation de l'accusé, la nature de l'infraction, surseoir au prononcé de la sentence et libérer l'accusé selon des conditions prescrites dans une ordonnance de probation ${ }^{i \overline{ }}$. D'autre part, la Commission nationale des libérations conditionnelles ${ }^{1: 8}$ pourra parfois accorder à un détenu une libération conditionnelle, sous réserve de certaines modalités qu'elle juge opportunes. Le milieu dans lequel sera plongé l'accusé ou le détenu à sa sortie de la cour ou de prison influera certainement sur la décision que sera amené à prendre le juge ou la Commission. On n'est pas sans savoir par exemple que ce sont les juges les plus soucieux de la réhabilitation des accusés qui se montrent parfois le plus sévère à leur égard; ils condamneront par exemple un jeune accusé à purger une peine de deux ans plutôt que de six mois au Centre fédéral de formation de façon à lui permettre d'apprendre un métier.

Dans ce que l'on a appelé l'affaire Koscott, une entreprise de ventes pyramidales, un très grand nombre de participants furent condamnés à des amendes variant entre $\$ 1000$ et $\$ 5000$ devant la Cour municipale de Montréal. Or, les chroniqueurs judiciaires ne manquèrent pas de souligner qu'un député créditiste à Ottawa, également impliqué dans cette affaire, fut le seul à bénéficier de la part du juge d'une ordonnance de libération inconditionnelle en vertu de l'article $662(1)$ du Code criminel et s'expliquaient difficilement cette clémence de la Cour ${ }^{\text {t: }}$.

Dans Choinière c. $R .^{\text {." }}$, l'appelante avait été condamnée à 3 ans de prison pour vol avec violence. Devant la Cour d'appel du Québec, elle réclamait un sursis de sentence vu qu'elle n'était âgée que de 20 ans. La Cour faisait remarquer :

There are factors in her case which encourage one to hope that there is a chance of rehabilitation, and if the court felt that releasing her to her family would assist in this it might be disposed to render judgment accordingly. There are, however, factors that make the court doubt that this is advisable. The court is told that her parents are poor but respectable people, but it is disturbed by the fact that not only did one of her brothers assist her in the crime but another was present in the same night club $^{i 2}$.

67. Art. 663 à 667 C. cr.

68. Loi sur la libération conditionnelle de détenus. S.R.C. 1970, c. P.2. Voir aussi : Rapport du groupe d'érude sur la mise en liberté des détenus, Ottawa, Information Canada. 1973.

69. La Presse, 1 juin 1973. p. A-6.

70. [1965] B.R. 714.

71. Id., p. 715 . 
C'est pourquoi elle croyait préférable de tenir l'appelante éloignée de son milieu familial et la meilleure façon d'y parvenir, pensait-elle, c'était de l'envoyer en prison.

Les critères sur lesquels se fonde la Commission nationale des libérations ne sont guère précis. L'article $10(1)$ de la Loi sur la libération conditionnelle des détenus ${ }^{72}$ parle de modalités que la Commission juge opportunes et spécifie qu'elle doit se demander si l'octroi d'une liberation conditionnelle "facilite le redressement et la réhabilitation du détenu».

La Commission exigera habituellement du détenu, entre autres, qu'il s'efforce de travailler régulièrement, qu'il évite de fréquenter des endroits et des gens de mauvaise réputation, qu'il obéisse à la loi et qu'il s'acquitte de toutes ses responsabilités légales et sociales. Cette dernière allégation est si vague qu'elle pourrait, semble-t-il, justifier la révocation de la libération conditionnelle dans presque n'importe quelle circonstance, et encore plus facilement dans le cas d'une personne vivant en culture de pauvreté. Il est évident qu'il est beaucoup plus difficile pour un pauvre, vu son milieu familial (où les parents s'intéressent peu à leurs enfants), le voisinage criminogène, ses aptitudes personnelles (tendance à la violence) et ses possibilités d'emploi, de répondre aux exigences de la Commission. Comme le soulignait Patricia Wald : "In the slum areas where life is lived on the streets and in the bars, where a sizeable percentage of local residents are past offenders lque ce soient des «bookies", des "pimps", des «dope pushers" ou autres parasites de la pauvreté], the conditions of probation may not be realistic. Probationers, like other slum-dwellers, probably have a greater chance of being "picked up" for a minor street offense because of where and how they live. Now because of their special status... they may attract even closer official attention in areas which police are trying to «keep clean ${ }^{73}$. Ainsi la personne riche, en raison de sa situation économique et de travail, de ses relations, de son lieu d'habitation, a beaucoup plus de chances d'obtenir son élargissement assez rapidement; c'est parfois le cas de ces professionnels du crime communément appelés par les mass media "hommes d'affaires". Peut-être faudrait-il accepter de prendre de plus grands «risques" à l'égard des personnes défavorisées si on ne veut pas les pénaliser et éviter de leur imposer des conditions qui touchent à des aspects de leur vie qui n'ont aucun rapport avec l'infraction commise?

72. S.R.C. 1970, c. P-2.

73. Wald, loc. cit., p. 292. 
Au Canada il a été clairement démontré que les délinquants autochtones bénéficient de moins de mises en liberté surveillée que les autres délinquants, tant dans les régions urbaines que rurales de la Saskatchewan et qu'il est pressant de nous orienter vers une augmentation des facilités de mise en liberté surveillée et de probation pour les délinquants autochtones'. D'autres études portant sur des groupes minoritaires défavorisés nous révèleraient probablement de semblables conclusions.

\section{CONCLUSION}

S'il y a un langage du pauvre, une psychologie du pauvre, une façon de voir du pauvre, il y a aussi une justice du pauvre. Être pauvre, c'est participer pour un grand nombre, à une culture radicalement différente de celle qui domine la société "normale», c'est s'adapter à cette société à sa façon, c'est être marginal. Il est un fait que la criminalité est intimement liée au phénomène de pauvreté. Mais les attitudes discriminatoires sinon répressives des différents acteurs de notre processus de justice pénale contribuent, jusqu'à un certain point, à grossir les chiffres de cette criminalité comparativement aux autres groupes de notre société. Les personnes des quartiers populaires sont effectivement plus souvent arrêtées et plus souvent condamnées à des peines relativement plus lourdes, et obtiennent de fait plus difficilement leur libération. Ce n'est pas que la loi en elle-même est toujours discriminatoire; c'est dans son application cependant que les choses prennent parfois une tournure bien différente.

Des réformes ont déjà été entreprises pour éliminer de notre droit les injustices les plus criantes et faciliter aux pauvres l'exercice de leurs droits devant nos tribunaux au même titre que les autres citoyens. Ces efforts sont certes très louables. Quoi qu'il en soit ce n'est que le jour où l'on s'attaquera aux véritables causes de la pauvreté et non simplement aux symptômes que l'on pourra penser à réduire considérablement la criminalité des milieux défavorisés. C'est de cette seule façon que l'on peut réellement réhabiliter un secteur important de notre population et l'intégrer à la société tout entière.

74. Schmeiser, Heumann et Manning, loc. cit. p. 73 à 78, 88. 